\title{
The variable spin-down rate of the transient magnetar XTE J1810-197
}

\author{
Fabio Pintore $^{1}$, Federico Bernardini ${ }^{2,12}$, Sandro Mereghetti ${ }^{1}$, Paolo Esposito ${ }^{1}$, \\ Roberto Turolla ${ }^{3,11}$, Nanda Rea ${ }^{4,5}$, Francesco Coti Zelati ${ }^{6,4,10}$, \\ Gian Luca Israel ${ }^{7}$, Andrea Tiengo ${ }^{8,1,9}$, Silvia Zane ${ }^{11}$ \\ ${ }^{1}$ INAF - IASF Milano, Via E. Bassini 15, I-20133 Milano, Italy \\ 2 New York University Abu Dhabi, Saadiyat Island, Abu Dhabi, 129188, United Arab Emirates \\ ${ }^{3}$ Dipartimento di Fisica e Astronomia, Universita di Padova, via F. Marzolo 8, I-35131 Padova, Italy \\ ${ }^{4}$ Anton Pannekoek Institute for Astronomy, University of Amsterdam, Postbus 94249, NL-1090-GE Amsterdam, the Netherlands \\ ${ }^{5}$ Instituto de Ciencias de l'Espacio (ICE, CSIC-IEEC), Carrer de Can Magrans, S/N, 08193, Barcelona, Spain \\ ${ }^{6}$ Universita dell'Insubria, via Valleggio 11, I-22100 Como, Italy \\ 7 INAF - Osservatorio Astronomico di Roma, via Frascati 33, I-00040 Monteporzio Catone, Roma, Italy \\ ${ }^{8}$ IUSS - Istituto Universitario di Studi Superiori, piazza della Vittoria 15, I-27100 Pavia, Italy \\ ${ }^{9}$ Istituto Nazionale di Fisica Nucleare, Sezione di Pavia, via A. Bassi 6, 27100 Pavia, Italy \\ ${ }^{10}$ INAF - Osservatorio Astronomico di Brera, Via Bianchi 46, I-23807 Merate (LC), Italy \\ ${ }^{11}$ MSSL, University College London, Holmbury St. Mary, Dorking Surrey, RH5 6NT, UK \\ 12 INAF - Osservatorio Astronomico di Capodimonte, Salita Moiariello 16, I-80131 Napoli, Italy
}

19 October 2018

\begin{abstract}
We have analyzed XMM-Newton and Chandra observations of the transient magnetar XTE J1810-197 spanning more than 11 years, from the initial phases of the 2003 outburst to the current quiescent level. We investigated the evolution of the pulsar spin period and we found evidence for two distinct regimes: during the outburst decay, $\dot{\nu}$ was highly variable in the range $-(2-4.5) \times 10^{-13} \mathrm{~Hz} \mathrm{~s}^{-1}$, while during quiescence the spin-down rate was more stable at an average value of $-1 \times 10^{-13} \mathrm{~Hz} \mathrm{~s}^{-1}$. Only during $\sim 3000$ days (from MJD 54165 to MJD 56908) in the quiescent stage it was possible to find a phase-connected timing solution, with $\dot{\nu}=-4.9 \times 10^{-14} \mathrm{~Hz} \mathrm{~s}^{-1}$, and a positive second frequency derivative, $\ddot{\nu}=1.8 \times 10^{-22} \mathrm{~Hz} \mathrm{~s}^{-2}$. These results are in agreement with the behavior expected if the outburst of XTE J1810-197 was due to a strong magnetospheric twist.
\end{abstract}

Key words: stars: magnetars - stars: neutron - X-rays: stars - magnetic fields - pulsars: individual: (XTE J1810-197)

\section{INTRODUCTION}

Magnetars are isolated neutron stars whose persistent emission and occasional outbursts are powered by magnetic energy (Duncan \& Thompson 1992, Thompson \& Duncan 1993, Paczynski 1992, see also Mereghetti 2008, Turolla et al. 2015). XTE J1810-197 was discovered with the Rossi X-ray Timing Explorer (RXTE) as a 5.45 s X-ray pulsar (Ibrahim et al. 2004) during a bright outburst in 2003, and associated to a previously known but unclassified ROSAT source. Further multiwavelength observations (Woods et al. 2005 Rea et al.2004, Halpern et al.|2008), led to classify XTE J1810-197 as a magnetar candidate.

XTE J1810-197 is the prototype of transient members of this class of sources. It likely spent at least 23 years in quiescence (at a flux of $\sim 7 \times 10^{-13} \mathrm{erg} \mathrm{s}^{-1} \mathrm{~cm}^{-2}$, in the $0.5-10 \mathrm{keV}$ energy band) before entering in outburst, in the 2003, when the flux increased by a factor of $\sim 100$ (Gotthelf et al. 2004). For an estimated distance of $3.5 \mathrm{kpc}$ (Camilo et al. 2006, Minter et al. 2008), the maximum observed luminosity was $\sim 10^{35} \mathrm{erg} \mathrm{s}^{-1}$, but XTE J1810-197 might have reached an even higher luminosity, since the initial part of the outburst was missed. XTE J1810-197 was also the first magnetar from which pulsed radio emission was detected (Camilo et al. 2006 2007). A large, unsteady spin-down of $\dot{P} \sim 10^{-11} \mathrm{~s} \mathrm{~s}^{-1}$ was measured during the outburst decay through radio and X-ray observations, which suggested that the surface dipolar magnetic field is $\sim 2 \times 10^{14} \mathrm{G}$ (Gotthelf et al. 2004, Ibrahim et al. 2004, Camilo et al. 2006).

The spectrum of XTE J1810-197 during the outburst has been modeled by several authors with two or three blackbody components of different temperature. The colder one has been interpreted 
Table 1. Log of the XMM-Newton and Chandra observations.

\begin{tabular}{|c|c|c|c|c|}
\hline $\begin{array}{l}\text { Obs. } \\
\text { No. }\end{array}$ & Satellite & Obs. ID & $\begin{array}{c}\text { Epoch }^{a} \\
\text { MJD }\end{array}$ & $\begin{array}{c}\text { Duration } \\
\text { ks }\end{array}$ \\
\hline 1 & Chandra & 4454 & 52878.9386632 & 4.3 \\
\hline 2 & XMM-Newton & 0161360301 & 52890.5595740 & 9.5 \\
\hline 3 & XMM-Newton & 0161360401 & 52890.7083079 & 2.1 \\
\hline 4 & XMM-Newton & 0152833201 & 52924.1677914 & 7.0 \\
\hline 5 & Chandra & 5240 & 52944.6289075 & 5.4 \\
\hline 6 & XMM-Newton & 0161360501 & 53075.4952632 & 17.2 \\
\hline 7 & $X M M$-Newton & 0164560601 & 53266.4995129 & 26.7 \\
\hline 8 & XMM-Newton & 0301270501 & 53447.9973027 & 40.0 \\
\hline 9 & XMM-Newton & 0301270401 & 53633.4453382 & 40.0 \\
\hline 10 & XMM-Newton & 0301270301 & 53806.7899360 & 41.8 \\
\hline 11 & Chandra & 6660 & 53988.8111877 & 31.8 \\
\hline 12 & XMM-Newton & 0406800601 & 54002.0627203 & 48.1 \\
\hline 13 & XMM-Newton & 0406800701 & 54165.7713547 & 60.2 \\
\hline 14 & XMM-Newton & 0504650201 & 54359.0627456 & 72.7 \\
\hline 15 & Chandra & 7594 & 54543.0034395 & 31.5 \\
\hline 16 & $X M M$-Newton & 0552800301 & 54895.5656089 & 4.3 \\
\hline 17 & $X M M$-Newton & 0552800201 & 54895.6543341 & 63.6 \\
\hline 18 & XMM-Newton & 0605990201 & 55079.6256771 & 19.4 \\
\hline 19 & XMM-Newton & 0605990301 & 55081.5548494 & 17.7 \\
\hline 20 & XMM-Newton & 0605990401 & 55097.7062563 & 12.0 \\
\hline 21 & Chandra & 11102 & 55136.6570779 & 26.5 \\
\hline 22 & Chandra & 12105 & 55242.6870526 & 15.1 \\
\hline 23 & Chandra & 11103 & 55244.7426533 & 14.6 \\
\hline 24 & $X M M$-Newton & 0605990501 & 55295.1863453 & 7.7 \\
\hline 25 & Chandra & 12221 & 55354.1368700 & 11.5 \\
\hline 26 & XMM-Newton & 0605990601 & 55444.6796630 & 9.1 \\
\hline 27 & Chandra & 13149 & 55494.1643981 & 16.8 \\
\hline 28 & Chandra & 13217 & 55600.9885520 & 16.2 \\
\hline 29 & XMM-Newton & 0671060101 & 55654.0878884 & 17.4 \\
\hline 30 & $X M M-N e w t o n$ & 0671060201 & 55813.3872852 & 13.7 \\
\hline 31 & Chandra & 13746 & 55976.3735837 & 22.5 \\
\hline 32 & Chandra & 13747 & 56071.3650797 & 22.1 \\
\hline 33 & $X M M$-Newton & 0691070301 & 56176.9826811 & 15.7 \\
\hline 34 & $X M M$-Newton & 0691070401 & 56354.1968379 & 15.7 \\
\hline 35 & XMM-Newton & 0720780201 & 56540.8584298 & 21.2 \\
\hline 36 & Chandra & 15870 & 56717.3097928 & 22.1 \\
\hline 37 & XMM-Newton & 0720780301 & 56720.9705351 & 22.7 \\
\hline 38 & Chandra & 15871 & 56907.9508362 & 21.7 \\
\hline
\end{tabular}

${ }^{a}$ Mean time of the observation.

as the (persistent) emission from the whole neutron star surface, while the hotter ones have been associated to cooling regions responsible for the outburst (Gotthelf et al. 2004, Bernardini et al. 2009 2011; Alford \& Halpern 2016). The appearance of hot spots could be due to the release of (magnetic) energy deep in the crust, or to Ohmic dissipation of back-flowing currents as they hit the star surface (Perna \& Gotthelf 2008, Albano et al. 2010, Beloborodov 2009. Pons \& Rea 2012). The X-ray pulse profile was energydependent and time-variable in amplitude, and it could be generally modelled by a single sinusoidal function (e.g. Ibrahim et al. 2004 Camilo et al. 2007, Bernardini et al.|2009, 2011, Alford \& Halpern 2016).

Here we report on the pulse period evolution of XTE J1810 197 exploiting the full set of XMM-Newton and Chandra X-ray observations carried out in the years 2003-2014 during the outburst decay and in the following quiescent period.

\section{OBSERVATIONS AND DATA REDUCTION}

We made use of 24 XMM-Newton and 14 Chandra observations of XTEJ1810-197 totalizing an exposure time of $\sim 830 \mathrm{ks}$ (see the $\log$ of observations in Table 1 .
The XMM-Newton data were reduced using SAS v. 14.0.0 and the most recent calibrations. We used the data obtained with the EPIC instrument, which consists of one pn camera and two MOS cameras. For each observation, we selected events with single and double pixel events (PATTERN $\leqslant 4$ ) for EPIC-pn and single, double, triple and quadruple pixel events for EPIC-MOS (PATTERN $\leqslant 12$ ). We set 'FLAG=0' so to exclude bad pixels and events coming from the CCD edge. The source and background events were extracted from $30^{\prime \prime}$ and $60^{\prime \prime}$ radius circular regions, respectively. Time intervals with high particle background were removed.

In three observations (7, 13 and 35) we found inconsistent values between the phases of the pulses derived (as described in the next Section) from the MOS and pn data. This is due to a known sporadic problem in the timing of EPIC-pn data, causing a shift of \pm 1 second in the times attributed to the counts. We identified the times at which the problems occurred and corrected the data by adding (or subtracting) 1 second to the photon time of arrival from the instant when the problem occurred (see Martin-Carrillo et al. 2012).

The Chandra observations were reduced using the CIAO v.4.7 software and adopting the standard procedures. Source events were extracted from a region of $20^{\prime \prime}$ radius around the position of XTE J1810-197 and background counts from a similar region close to the source.

Photon arrival times of both satellites were converted to the Solar system barycenter using the milliarcsec radio position of XTE J1810-197 $(\mathrm{RA}=272.462875 \mathrm{deg}$, Dec. $=-19.731092 \mathrm{deg}$, (J2000); Helfand et al. 2007) and the JPL planetary ephemerides DE405.

\section{TIMING ANALYSIS}

In order to study the evolution of the spin frequency from outburst to quiescence (i.e. covering the whole data set) we initially measured the spin frequency in each individual pointing by applying a phase-fitting technique in every observation. The phase of a pulse is defined as $\phi=\phi_{0}+\int \nu d t$, where $\nu$ is the spin frequency. If the coherence of the signal is maintained between subsequent observations, the data can be be fitted by the polynomial:

$$
\phi(t)=\phi_{0}+\nu_{0}\left(t-T_{0}\right)+\frac{1}{2} \dot{\nu}\left(t-T_{0}\right)^{2}+\frac{1}{6} \ddot{\nu}\left(t-T_{0}\right)^{3}+\ldots
$$

where $T_{0}$ is the reference epoch, $\nu_{0}$ is the frequency at $T_{0}, \dot{\nu}$ is the spin frequency derivative and $\ddot{\nu}$ is second-order spin frequency derivative (e.g. Dall'Osso et al. 2003 for more details).

Thanks to the large counting statistics of each single observation, it was possible to obtain accurate measurements of the frequencies by applying the phase-fitting technique to a number of short time intervals (durations from $300 \mathrm{~s}$ to $5 \mathrm{ks}$, depending on the counting statistics) within each observation and we were able to align the pulse-phases by use only the linear term of Eq. 1 The frequencies derived in this way are plotted as a function of time in the middle panel of Figure 1], while in the top panel we show the flux evolution of XTE J1810-197.

To derive the fluxes plotted in Figure 1 we fitted the timeaveraged spectra of each observation with a model consisting of two to three blackbodies (see e.g. Bernardini et al. 2009, Alford \& Halpern 2016 for more details). The interstellar absorption was kept fixed to the value of $5.7 \times 10^{21} \mathrm{~cm}^{-2}$, derived from the spectrum of the first $X M M-N e w t o n$ observation. The temperatures that 


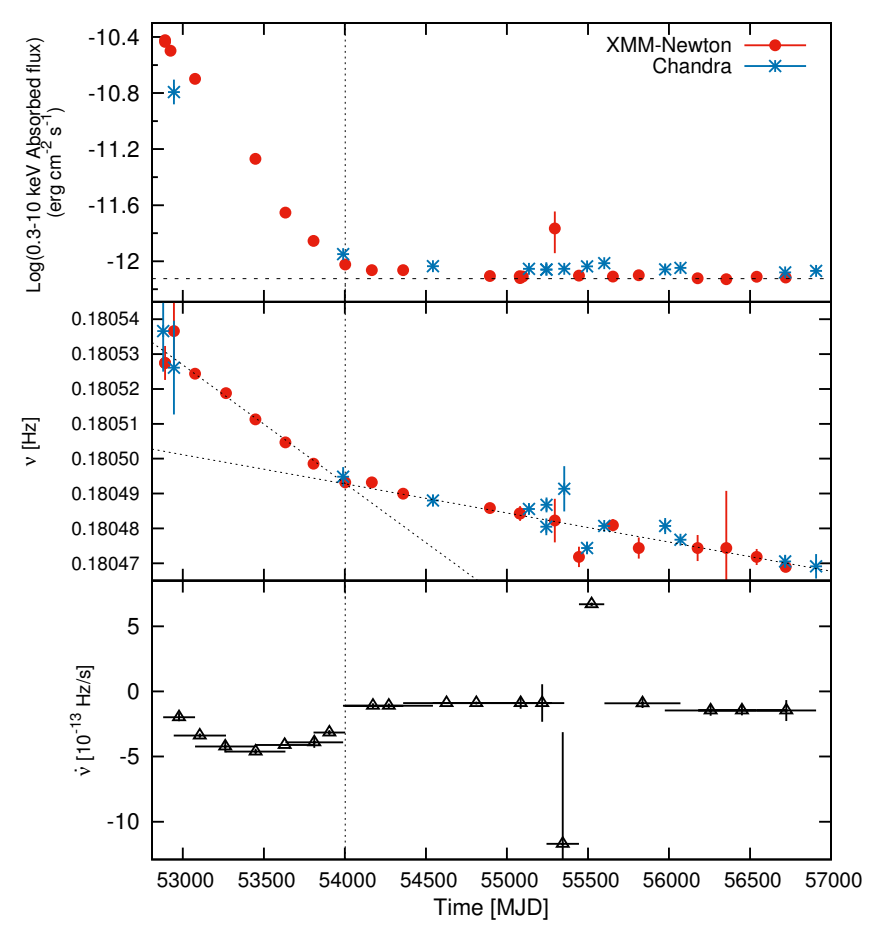

Figure 1. Top panel: evolution of the logarithmic absorbed flux in the 0.3$10 \mathrm{keV}$ energy range. The dashed line is the linear fit to the data after MJD 54500. The errors are at $90 \%$ confidence level. Center panel: spin frequency along the outburst of XTEJ1810-197 as found in the single observations. The dashed lines indicate the fits with two linear functions to the data before and after MJD 54000. Bottom panels: frequency derivatives as measured by linear fits of small sub-set of observations. The horizontal error bars indicate the time interval spanned by the observations used in each fit.

The vertical, dashed line indicates the epoch after which is possible to phase-connect the data. Errors in the center and bottom panels are at $1 \sigma$.

we found for the three blackbodies $(\sim 0.1,0.3$ and $0.5 \mathrm{keV})$ are consistent with those reported in Bernardini et al. (2009) and Alford \& Halpern (2016), to which we refer for more details. The maximum flux observed by XMM-Newton during the outburst was $(3.18 \pm 0.04) \times 10^{-11} \mathrm{erg} \mathrm{cm}^{-2} \mathrm{~s}^{-1}$ (absorbed flux in the 0.3-10 keV energy range). The flux decreased until about MJD 54500, after which it remained rather constant (see also Gotthelf \& Halpern 2007, Bernardini et al. 2011, Alford \& Halpern 2016). We found that the flux slowly decreased, finally reaching a constant value of $(7.5 \pm 0.2) \times 10^{-13} \mathrm{erg} \mathrm{cm}^{-2} \mathrm{~s}^{-1}$, which we derived by fitting with a constant the fluxes of all the observations after MJD 54500 (see Fig.1 1top panel). This value is within the range of fluxes measured by ROSAT, ASCA and Einstein before the onset of the outburst $\left((5-10) \times 10^{-13} \mathrm{erg} \mathrm{cm}^{-2} \mathrm{~s}^{-1}\right.$; Gotthelf et al. 2004).

It is clear from Fig. 1 that the source timing properties tracked remarkably well the evolution of the flux. The average spin-down rate was larger during the first 3-4 years, during the outburst decay, and then it decreased while the source was in (or close to) quiescence. We can distinguish two time intervals, separated at MJD $\sim 54000$, in which a linear fit can approximately describe the frequency evolution. The slopes of the two linear functions are $(-3.9 \pm 0.2) \times 10^{-13} \mathrm{~Hz} \mathrm{~s}^{-1}\left(\chi_{\nu}^{2} /\right.$ dof $\left.=6.7 / 9\right)$ and $(-1.00 \pm 0.05) \times 10^{-13} \mathrm{~Hz} \mathrm{~s}^{-1}\left(\chi_{\nu}^{2} /\right.$ dof $\left.=1.8 / 24\right)$ before and after MJD 54000, respectively. These values represent the longterm averaged spin-down rates, but the residuals of the linear fits indicate that the time evolution of the frequency derivative is more complex. To better investigate this behavior, we performed several linear fits to small groups of consecutive frequency measurements. We adopted a moving-window approach by using partially overlapping sets of points. In this way we obtained the $\dot{\nu}$ values plotted in the bottom panel of Figure 1 They show a highly variable spindown rate, especially during the outburst decay, when it ranged from $-4.5 \times 10^{-13} \mathrm{~Hz} \mathrm{~s}^{-1}$ to $-0.5 \times 10^{-13} \mathrm{~Hz} \mathrm{~s}^{-1}$.

Phase-coherent timing solutions for XTEJ1810-197 have been reported for the initial part of the outburst (Ibrahim et al.2004, Camilo et al. 2007). We tried to phase-connect all the XMM-Newton and Chandra observations, but this turned out to be rather difficult due to the large timing noise. However, we were able to find a phase-connected solution for the data during quiescence (i.e. all the observations obtained after MJD 54100), as follows. For each observation, we folded the EPIC (pn plus MOS) or Chandra data at a frequency of $0.18048 \mathrm{~Hz}$ (corresponding to $P=5.54078 \mathrm{~s}$, the average spin period after MJD 54100). For each observation the phase of the pulsation was then derived by fitting a constant plus a sinusoid to the folded pulse profile in the $0.3-10 \mathrm{keV}$ energy range. We initially aligned, with only the linear term in Eq. 1 the XMM-Newton observations 18 and 19 that were the most closely spaced ( $\sim 2$ days). Then, we included one by one the other observations, as the uncertainty on the best-fit parameters became increasingly smaller allowing us to connect more distant points. We included higher order derivatives only if the improvement in the fit was significant in the timing solution. After the inclusion of Chandra observations 21 and 22, the quadratic term became statistically significant, while the third order polynomial term was needed after the inclusion of observations 25 and 26 . The best fit parameters of the final solution are reported in Table 2 and the fit is shown in Figure 4 The fit with $\nu, \dot{\nu}$ and $\ddot{\nu}$ has $\chi_{\nu}^{2}=65.7$ (for 20 dof). Such a large value reflects the presence of a strong timing noise. In fact, the residuals shown in the lower panel of Figure 4 indicate significant deviations from the best fit solution, especially during the last 1000 days, when they are as large as $\sim 0.2$ cycles in phase.

Some timing irregularity occurred also when the source was in quiescence. In particular, around MJD 55400 the spin-down rate was much larger than the quiescent average value and larger than that seen during the outburst decay. Quite remarkably, also a spin-up episode was detected (see Figure 1-bottom). This is better illustrated in Figure 3 which shows the frequency measurements around this time. Unfortunately, the sparse coverage and the large error bars of some points do not allow us to establish whether this was a sudden event, like an anti-glitch, or simply due to an increased timing noise episode. Assuming that the time irregularity is an anti-glitch, we fitted the data in the time range MJD 5430057000, with the following simple model:

$$
\begin{array}{ll}
\nu(t)=\nu_{0}+\dot{\nu_{0}} \cdot t & \text { for } t<t_{g} \\
\nu(t)=\nu_{0}+\dot{\nu_{0}} \cdot t+\Delta \nu \cdot e^{\left(-\left(t-t_{g}\right) / \tau\right)} & \text { for } t>t_{g}
\end{array}
$$

where $\tau$ is the decay time and $t_{g}$ is the time of the glitch, which we kept fixed in the fit. If the glitch occurred immediately after observation $25\left(t_{g}=55354\right)$, we obtained a good fit $\left(\chi_{\nu}^{2}=1.14\right.$ for 21 dof, shown by the solid line in Figure 3 with $\Delta \nu=(6.5 \pm$ $5.8) \times 10^{-5} \mathrm{~Hz}, \tau=51 \pm 21$ days, $\nu_{0}=0.18093(3) \mathrm{Hz}$ and $\dot{\nu_{0}}=-9.4(3) \times 10^{-14} \mathrm{~Hz} \mathrm{~s}^{-1}$. If instead the glitch occurred at observation $26\left(t_{g}=55444\right)$, we obtain $\Delta \nu<1 \times 10^{-4} \mathrm{~Hz}$ and $\tau<200$ days ( $3 \sigma$ upper limits). 


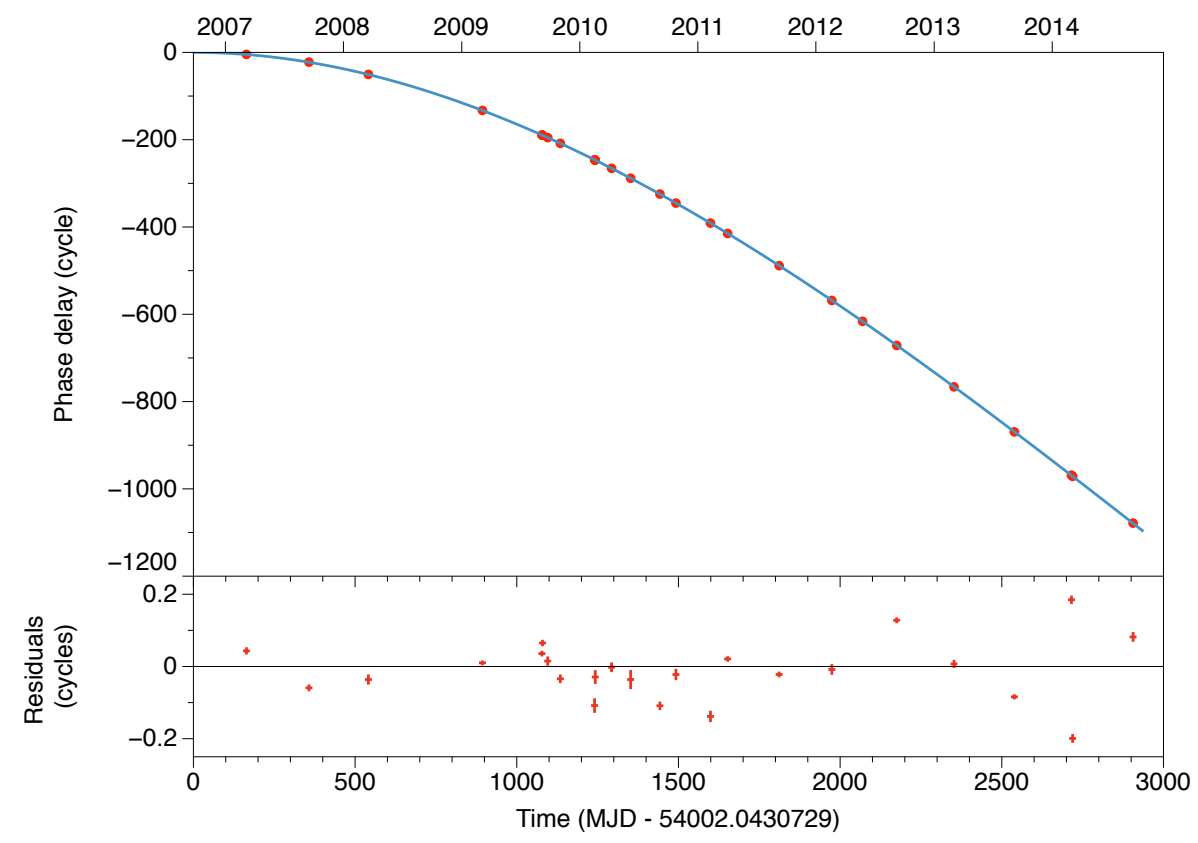

Figure 2. Phase-connection of $\sim 3000$ days of XMM-Newton and Chandra data (observations from 13 to 38) using a third order polynomial function. Top: the red points are the measured phases, one for each observation, and the solid line is the best fit model; bottom: residuals with respect to the best fit model.

Table 2. Best-fit timing solution of the XMM-Newton and Chandra observations. Errors are at $1 \sigma$.

\begin{tabular}{lcc}
\hline Parameter & & Units \\
\hline Time range & $54165-56908$ & $\mathrm{MJD}$ \\
$\mathrm{T}_{0}{ }^{a}$ & 54002.0430729 & $\mathrm{MJD}$ \\
$\nu_{0}$ & $0.1804821(1)$ & $\mathrm{Hz}$ \\
$\dot{\nu}$ & $-4.9(2) \times 10^{-14}$ & $\mathrm{~Hz} \mathrm{~s}^{-1}$ \\
$\ddot{\nu}$ & $1.8(1) \times 10^{-22}$ & $\mathrm{~Hz} \mathrm{~s}^{-2}$ \\
$P$ & $5.540716(3)$ & $\mathrm{s}$ \\
$\dot{P}$ & $1.51(7) \times 10^{-12}$ & $\mathrm{~s} \mathrm{~s}^{-1}$ \\
$\ddot{P}$ & $-5.5(4) \times 10^{-21}$ & $\mathrm{~s} \mathrm{~s}^{-2}$ \\
$\chi_{\nu}^{2}($ dof $)$ & $65.7(20)$ & \\
\hline
\end{tabular}

${ }^{a}$ Reference epoch.

\section{DISCUSSION}

Variations in the spin-down rate are not uncommon in magnetars and have been observed both in transient and persistent sources. They are believed to originate from changes in the magnetosphere geometry and particles outflow which produce a varying torque on the neutron star. Since also the emission properties from magnetars depend on the evolution of their dynamic magnetospheres, some correlation between spin-period evolution and radiative properties is not surprising.

The most striking examples, among persistent magnetars, are given by SGR 1806-20 and 1E 1048.1-5937. The average spindown rate of SGR 1806-20, as well as its spectral hardness, increased in the $\sim 4$ years of enhanced bursting activity which led to the giant flare of December 2004 (Mereghetti et al.|2005). However, a further increase (by a factor of 2-3) of the long term spindown rate occurred both in 2006 and 2008, while the flux and bursting rate showed no remarkable changes (Younes et al. 2015). In 1E 1048.1-5937, significant enhancements of the spin-down rate, which then subsided through repeated oscillations, have been ob-

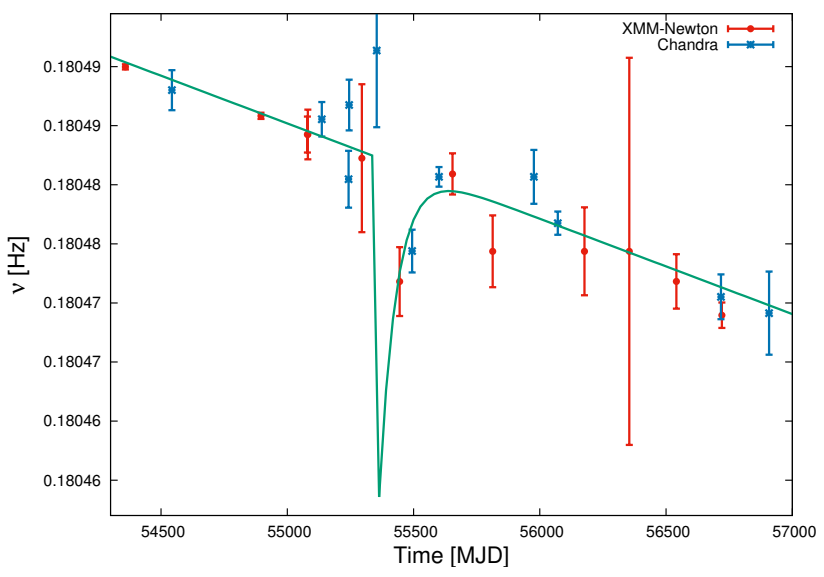

Figure 3. Frequencies measured around the time of the possible anti-glitch. The solid line is the best-fit discussed in the text.

served to lag the occurrence of X-ray outbursts (Archibald et al. 2015). Other persistent magnetars, for which phase-coherent timing solutions extending over several years could be mantained, showed $\dot{\nu}$ variations and/or glitches, sometimes (but not always) related to changes in the source flux and the emission of bursts (e.g. Dib \& Kaspi 2014).

Transient magnetars offer, in principle, the best opportunity to investigate the correlations between the variations in the spindown rate and the radiative properties. However, the observations of transient magnetars carried out up to now have shown a variety of different behaviors. Furthermore, for many of them, no detailed information is available on the spin-down during the quiescent state, that instead in this work we now have found for XTE J1810-197 . No firm conclusion on the evolution of the spin-down rate could be derived from the two outbursts of CXOU J164710.2-455216, for which a positive $\ddot{\nu}$ was reported only during the decay of the 
first outburst, while the insufficient time coverage prevented such a measure for the second one (Rodríguez Castillo et al.|2014). A positive $\ddot{\nu}$ was reported for both Swift J1822.3-1606 (which went in outburst in July 2011 and was subsequently monitored for about 500 days; Rodríguez Castillo et al. 2015), as well as for SGR J1935+2154 (outburst in July 2014, time coverage $\sim 260$ days; Israel et al. 2016), and, tentatively, also for SGR 0501+4516 (for this source observations actually covered part of the quiescent state but phase connection along the entire dataset could not be ensured; Camero et al. 2014). On the other hand, an increase of the spindown rate during the outburst decay was reported for SGR J174529 (Kaspi et al. 2014, Coti Zelati et al. 2015).

Our analysis of XMM-Newton and Chandra data spanning 11 years has shown that in the transient magnetar XTE J1810-197 the spin frequency evolution tracked remarkably well the luminosity state. During the outburst decay, the average spin-down rate was $(-3.9 \pm 0.2) \times 10^{-13} \mathrm{~Hz} \mathrm{~s}^{-1}$, but large variations around this value were seen, as already noticed by several authors (Halpern \& Gotthelf 2005, Camilo et al. 2007, Bernardini et al. 2009). During the long quiescent state after the end of the outburst, the average spindown rate was a factor of $\sim 4$ smaller. Although some timing noise was still present, the variations in $\dot{\nu}$ were smaller in the quiescent state, except for a few months in Summer 2010. The timing irregularities in that period might have been caused by the occurrence of an anti-glitch, similar to that seen in the persistent magnetar $1 \mathrm{E}$ 2259+586 (Archibald et al. 2013). We found that the pulse-shape in the $0.3-10 \mathrm{keV}$ energy range was nearly sinusoidal and the pulse fraction decreased during the outburst decay, as already reported by e.g. Perna \& Gotthelf (2008), Albano et al. (2010) and Bernardini et al. (2009). We note that the pulse-shape remained nearly sinusoidal also during quiescence (see also Bernardini et al.2011, Alford \& Halpern 2016).

The spectral properties of magnetars are commonly explained in terms of the twisted magnetosphere model (Thompson et al. 2002), according to which part of the magnetic helicity is transferred from the internal to the external magnetic field, which acquires a non-vanishing toroidal component (a twist). The currents required to support the twisted external field resonantly up-scatter thermal photons emitted by the star surface, leading to the formation of the power-law tails observed up to hundreds of keV. Since twisted fields have a weaker dependence on the radial distance with respect to a dipole, the higher magnetic field at the light cylinder radius results in an enhanced spin-down rate. The increased activity of magnetars is often associated to the development (or an increase) of a twist, which should lead to higher fluxes, local surface temperature increases, harder spectra and larger spin-down rates. However, this holds for globally twisted fields (meaning that the twist affects the entire external field). The transport of helicity from the interior is mediated by the star crust: in order to occur the crust must yield, allowing a displacement of the field lines. Crustal displacements are small compared to the star radius, so the twist is most likely localized to a bundle of field lines anchored on the displaced platelet (Beloborodov 2009). Once implanted, the twist must necessarily decay to maintain its own supporting currents, unless energy is constantly supplied from the star interior. The sudden appearance of a localized twist and its subsequent decay can explain some of the observed properties of transient magnetars (Beloborodov 2009. Albano et al. 2010), including the fact that transient spectra are often thermal, as in the case of XTE J1810-197, since resonant Compton scattering may be not very effective, although the mechanism responsible for the heating of the star surface is still unclear (either Ohmic dissipation by backflowing currents or deep crustal heating; Beloborodov 2009, Pons \& Rea 2012). If strong enough, a localized twist can still influence the spin-down rate, which is expected to increase first and then decrease as the magnetosphere untwists, as we observed in XTE J1810-197.

\section{CONCLUSIONS}

XTE J1810-197 was the first transient magnetar to be discovered and it is probably one of the best studied. In particular, it has been possible to trace in great detail its spectral properties over the long ( $\sim 3$ years) outburst decay and to monitor it during quiescence for several years afterwards. By investigating the evolution of its spin frequency with all the available XMM-Newton and Chandra data, we found evidence for two distinct regimes: during the outburst decay, $\dot{\nu}$ was highly variable in the range $-(2-4.5) \times 10^{-13} \mathrm{~Hz}$ $\mathrm{s}^{-1}$, while during quiescence the spin-down rate was more stable and had an average value smaller by a factor $\sim 4$.

This evolution of the spin-down rate is in agreement with the suggestion that the outburst of transient magnetars may be caused by a strong twist of a localized bundle of magnetic field lines ( $\mathrm{Be}-$ loborodov 2009). Evidences for an evolution of $\dot{\nu}$ in other transient magnetars are far less conclusive, possibly reflecting the fact that, if the twist is not very strong, or the twisted bundle too localized, its effect on the spin-down rate are smaller. A detailed calculation of the spin-down torque for a spatially-limited twisted field requires a full non-linear approach and has not been presented yet. Beloborodov (2009) discussed a simple estimate, valid for small twists $(\psi<1 \mathrm{rad})$

$$
\Delta \mu / \mu \sim\left(\psi^{2} / 4 \pi\right) \log \left(u_{*} / u_{\mathrm{LC}}\right),
$$

where $\Delta \mu$ is the "equivalent" increase in the dipole moment produced by the twist and $u$ is the area of the j-bundle, evaluated at the star surface and at the light cylinder. Since $\Delta \dot{\nu} / \nu \sim 2 \Delta \mu / \mu$, a fractional variation of $\dot{\nu}$ of a factor of $\sim 4$, as observed (see Figure 1 lower panel), can not be achieved with a small twist, $\psi<1$. This indicates that the (maximal) twist in XTE J1810-197 was most probably larger, $\psi \gtrsim 1 \mathrm{rad}$, so that equation 2 does not hold anymore. A quite large value of the twist in the outburst of XTE J1810 197 was also inferred by Beloborodov (2009), on the (qualitative) basis that only a strong twist can produce a change of the spin-down rate.

\section{REFERENCES}

Albano A., Turolla R., Israel G. L., Zane S., Nobili L., Stella L., 2010, ApJ, 722, 788

Alford J., Halpern J., 2016, ArXiv e-prints

Archibald R. F., Kaspi V. M., Ng C.-Y., Gourgouliatos K. N., Tsang D., Scholz P., Beardmore A. P., Gehrels N., Kennea J. A., 2013, Nature, 497, 591

Archibald R. F., Kaspi V. M., Ng C.-Y., Scholz P., Beardmore A. P., Gehrels N., Kennea J. A., 2015, ApJ, 800, 33

Beloborodov A. M., 2009, ApJ, 703, 1044

Bernardini F., Israel G. L., Dall'Osso S., Stella L., Rea N., Zane S., Turolla R., Perna R., Falanga M., Campana S., Götz D., Mereghetti S., Tiengo A., 2009, A\&A, 498, 195

Bernardini F., Perna R., Gotthelf E. V., Israel G. L., Rea N., Stella L., 2011, MNRAS, 418, 638

Camero A., Papitto A., Rea N., Viganò D., Pons J. A., Tiengo A., Mereghetti S., Turolla R., Esposito P., Zane S., Israel G. L., Götz D., 2014, MNRAS, 438, 3291 
Camilo F., Ransom S. M., Halpern J. P., Reynolds J., 2007, ApJ, 666, L93

Camilo F., Ransom S. M., Halpern J. P., Reynolds J., Helfand D. J., Zimmerman N., Sarkissian J., 2006, Nature, 442, 892

Coti Zelati F., Rea N., Papitto A., Viganó D., Pons J. A., Turolla R., Esposito P., Haggard D., Baganoff F. K., Ponti G., et al. I., 2015, MNRAS, 449, 2685

Dall'Osso S., Israel G. L., Stella L., Possenti A., Perozzi E., 2003, ApJ, 599, 485

Dib R., Kaspi V. M., 2014, ApJ, 784, 37

Duncan R. C., Thompson C., 1992, ApJ, 392, L9

Gotthelf E. V., Halpern J. P., 2007, Ap\&SS, 308, 79

Gotthelf E. V., Halpern J. P., Buxton M., Bailyn C., 2004, ApJ, 605,368

Halpern J. P., Gotthelf E. V., 2005, ApJ, 618, 874

Halpern J. P., Gotthelf E. V., Reynolds J., Ransom S. M., Camilo F., 2008, ApJ, 676, 1178

Helfand D. J., Chatterjee S., Brisken W. F., Camilo F., Reynolds J., van Kerkwijk M. H., Halpern J. P., Ransom S. M., 2007, ApJ, 662,1198

Ibrahim A. I., Markwardt C. B., Swank J. H., Ransom S., Roberts M., Kaspi V., Woods P. M., Safi-Harb S., Balman S., Parke W. C., Kouveliotou C., Hurley K., Cline T., 2004, ApJ, 609, L21

Israel G. L., Esposito P., Rea N., Coti Zelati F., Tiengo A., Campana S., Rodriguez Castillo S. M. G. A., Gotz D., Burgay M., Possenti A., Zane S., Turolla R., 2016, ArXiv e-prints

Kaspi V. M., Archibald R. F., Bhalerao V., Dufour F., Gotthelf E. V., An H., Bachetti M., Beloborodov A. M., Boggs S. E., et al. C., 2014, ApJ, 786, 84

Martin-Carrillo A., Kirsch M. G. F., Caballero I., Freyberg M. J., Ibarra A., Kendziorra E., Lammers et al. 2012, A\&A, 545, A126 Mereghetti S., 2008, A\&A Rev., 15, 225

Mereghetti S., Götz D., von Kienlin A., Rau A., Lichti G., Weidenspointner G., Jean P., 2005, ApJ, 624, L105

Minter A. H., Camilo F., Ransom S. M., Halpern J. P., Zimmerman N., 2008, ApJ, 676, 1189

Paczynski B., 1992, Acta Astronomica, 42, 145

Perna R., Gotthelf E. V., 2008, ApJ, 681, 522

Pons J. A., Rea N., 2012, ApJ, in press (astro-ph/1203.4506)

Rea N., Testa V., Israel G. L., Mereghetti S., Perna R., Stella L., Tiengo A., Mangano V., Oosterbroek T., Mignani R., Lo Curto G., Campana S., Covino S., 2004, A\&A, 425, L5

Rodríguez Castillo G. A., Israel G. L., Esposito P., Pons J. A., Rea N., Turolla R., Viganò D., Zane S., 2014, MNRAS, 441, 1305

Rodríguez Castillo G. A., Israel G. L., Tiengo A., Salvetti D., Turolla R., Zane S., Rea N., Esposito P., Mereghetti S., Perna R., Stella L., Pons J. A., Campana S., Götz D., Motta S., 2015, ArXiv e-prints

Thompson C., Duncan R. C., 1993, ApJ, 408, 194

Thompson C., Lyutikov M., Kulkarni S. R., 2002, ApJ, 574, 332

Turolla R., Zane S., Watts A. L., 2015, Reports on Progress in Physics, 78, 116901

Woods P. M., et al., 2005, ApJ, 629, 985

Younes G., Kouveliotou C., Kaspi V. M., 2015, ApJ, 809, 165

\section{ERRATUM: "THE VARIABLE SPIN-DOWN RATE OF THE TRANSIENT MAGNETAR XTE J1810-197”}

Prompted by the recent paper by Camilo et al. (2016), we reexamined our phase-connected timing solution for XTE J1810-197
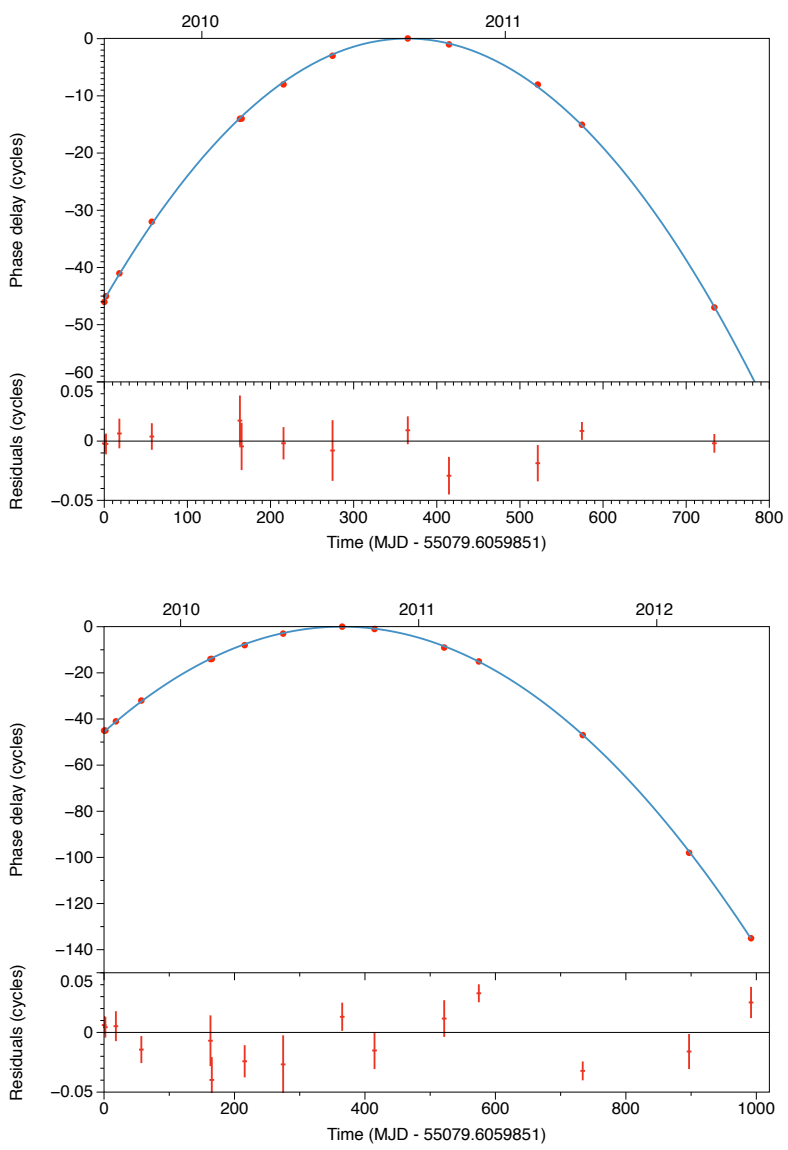

Figure 4. Phase-connection of $\sim 800$ (left) and $\sim 1000$ (right) days of $X M M-N e w t o n$ and Chandra data using a third order polynomial function. Top panels: the red points are the measured phases, one for each observation, and the solid line is the best fit model; bottom panels: residuals with respect to the best-fit model.

(Pintore et al. 2016), and we found a flaw in the procedure to compute the errors during some steps of our analysis. Due to this mistake, the phase-connected solution on 3000 days of X-ray data (reported in Tab. 2 and Fig. 2 of Pintore et al. 2016) is wrong.

With the new analysis of the data, we can phase-connect 13 observations with a good fit $\left(\chi_{\nu}^{2}(\right.$ dof $)=0.9(9)$; solution 1 in Tab.3 and Fig.4-top), from MJD 55079 to MJD 55814 (observations from 18 to 30 of Pintore et al. 2016). The inclusion of also the two observations at MJD 55976 and MJD 56071 (observations 31 and 32) yields best fit parameters (solution 2 in Tab.3 and Fig.4-bottom) consistent with those obtained by Camilo et al. (2016) for the same set of observations, but with a higher $\chi_{\nu}^{2}$ with respect to solution 1 .

The table and figures reported here supersede Tab. 2 and Fig. 2 of Pintore et al. (2016). We note that these changes do not affect the main conclusions of that paper.

\section{REFERENCES}

Camilo F., Ransom S. M., Halpern J. P., Alford J. A. J., Cognard I., Reynolds J. E., Johnston S., Sarkissian J., van Straten W., 2016, ApJ, 820, 110

Pintore F., Bernardini F., Mereghetti S., Esposito P., Turolla R., Rea N., Coti Zelati F., Israel G. L., Tiengo A., Zane S., 2016, MNRAS, 458,2088 
Table 3. Best-fitting timing solutions for 13 (solution 1) and 15 (solution 2) $X M M-N e w t o n$ and Chandra observations. Errors are at $1 \sigma$.

\begin{tabular}{lcc}
\hline Parameter & Solution 1 & Solution 2 \\
\hline Time range (MJD) & $55079-55814$ & $55079-56071 \mathrm{MJD}$ TDB \\
$\mathrm{T}_{0}{ }^{a}(\mathrm{MJD}$ TDB $)$ & 55444.0 & 55444.0 \\
$\nu_{0}(\mathrm{~Hz})$ & $0.18048121335(44)$ & $0.18048121599(27)$ \\
$\dot{\nu}\left(\mathrm{Hz} \mathrm{s}^{-1}\right)$ & $-9.2059(16) \times 10^{-14}$ & $-9.2085(16) \times 10^{-14}$ \\
$\ddot{\nu}\left(\mathrm{Hz} \mathrm{s}^{-2}\right)$ & $5.7(3) \times 10^{-23}$ & $3.80(13) \times 10^{-23}$ \\
$P\left(\mathrm{~s}^{-1}\right.$ & $5.540742892(14)$ & $5.540742811(8)$ \\
$\dot{P}\left(\mathrm{~s} \mathrm{~s}^{-1}\right)$ & $2.8262(5) \times 10^{-12}$ & $2.8270(5) \times 10^{-12}$ \\
$\ddot{P}\left(\mathrm{~s} \mathrm{~s}^{-2}\right)$ & $-1.75(9) \times 10^{-21}$ & $-1.16(4) \times 10^{-21}$ \\
$\chi_{\nu}^{2}(\mathrm{dof})$ & $0.9(9)$ & $5.0(11)$ \\
\hline
\end{tabular}

${ }^{a}$ Reference epoch. 